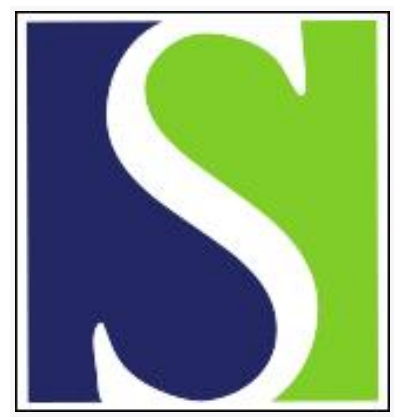

Scand J Work Environ Health 2015;41(2):164-174

https://doi.org/10.5271/sjweh.3478

Published online: 15 Jan 2015, Issue date: 01 Mar 2015

Log in and breathe out: internet-based recovery training for sleepless employees with work-related strain - results of a randomized controlled trial

by Thiart H, Lehr D, Ebert DD, Berking M, Riper H

The present study evaluates a newly developed, internet-based recovery training that considers the particular situation of employees experiencing both work stress and sleep problems. The results suggest that this low-threshold intervention could help a large number of employees overcome a deficient recovery from work.

Affiliation: Innovation Incubator, Division of Health Training Online, Leuphana University Lueneburg, Rotenbleicher Weg 67, 21335 Lueneburg, Germany. thiart@inkubator.leuphana.de

Refers to the following texts of the Journal: 2006;32(6):482-492 2006;32(6):493-501 2012;38(3):238-246 2013;39(6):535-549 $2014 ; 40(6): 582-596$

The following articles refer to this text: 2016;42(5):382-394; 2018;44(2):171-182; 2019;45(6):560-576; 2020;46(1):1-4

Key terms: CBT-I; cognitive behavioral therapy for insomnia; guided self-help; insomnia; internet; internet-based training; occupational health; online; randomized controlled trial; recovery; recovery training; recreation; sleepless; sleepless employee; stress; teacher

This article in PubMed: www.ncbi.nlm.nih.gov/pubmed/25590336

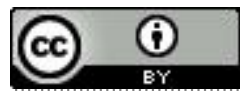




\title{
Log in and breathe out: internet-based recovery training for sleepless employees with work-related strain - results of a randomized controlled trial
}

\author{
by Hanne Thiart, MSc, ${ }^{1,2}$ Dirk Lehr, PhD, , 2 David Daniel Ebert, PhD, , 3 Matthias Berking, PhD, ${ }^{2,}{ }^{4}$ Heleen \\ Riper, $P h D^{2,5}$
}

\begin{abstract}
Thiart H, Lehr D, Ebert DD, Berking M, Riper H. Log in and breathe out: internet-based recovery training for sleepless employees with work-related strain - the results of a randomized controlled trial. Scand J Work Environ Health. 2015;41(2):164-174. doi:10.5271/sjweh.3478
\end{abstract}

\begin{abstract}
Objectives The primary purpose of this randomized controlled trial (RCT) was to evaluate the efficacy of a guided internet-based recovery training for employees who suffer from both work-related strain and sleep problems (GET.ON Recovery). The recovery training consisted of six lessons, employing well-established methods from cognitive behavioral therapy for insomnia (CBT-I) such as sleep restriction, stimulus control, and hygiene interventions as well as techniques targeted at reducing rumination and promoting recreational activities.
\end{abstract}

Methods In a two-arm RCT ( $\mathrm{N}=128)$, the effects of GET.ON Recovery were compared to a waitlist-control condition (WLC) on the basis of intention-to-treat analyses. German teachers with clinical insomnia complaints (Insomnia Severity Index $\geq 15$ ) and work-related rumination (Irritation Scale, cognitive irritation subscale $\geq 15$ ) were included. The primary outcome measure was insomnia severity.

Results Analyses of covariance (ANCOVA) revealed that, compared to the WLC, insomnia severity of the intervention group decreased significantly stronger $(\mathrm{F}=74.11, \mathrm{P}<0.001)$ with a $\mathrm{d}=1.45[95 \%$ confidence interval $(95 \% \mathrm{CI}) 1.06-1.84]$ The number needed to treat (NNT) was $<2$ for reliable change and NNT $<4$ for reduction in expert-rated diagnosis of primary insomnia.

Conclusion The training significantly reduces sleep problems and fosters mental detachment from work and recreational behavior among adult stressed employees at post-test and 6-months follow up. Given the low threshold access this training could reach out to a large group of stressed employees when results are replicated in other studies.

Key terms cognitive behavioral therapy for insomnia; CBT-I; guided self-help; insomnia; internet-based training; occupational health; online; recreation; stress; teacher.

There is growing evidence of the negative effects of work stress on sleep (1). Problems with restorative sleep are common: chronic insomnia has a $10 \%$ prevalence rate in Western industrialized countries (2-4). Impaired sleep is associated with personal distress, diminished general functioning, increased absenteeism and presenteeism and, in turn, high personal and societal costs (5-7). To effectively target sleep problems in a population of stressed employees, it is important to consider recovery research findings. Recovery from work is regarded as an explanatory mechanism in the relation between acute stress reactions and adverse health. It can be defined as the restoration of depleted resources by psychophysi- ological unwinding after work (8). Recovery from work is well recognized as an essential daily process to balance the strains of work and prevent general ill health (9-10).

There is evidence that successful recovery from work implies three core components which are closely interrelated: (i) restorative sleep which can be perceived as a particularly important process in recovery from work stress because basic resources are restored during sleep, and fatigue subsequently decreases (11-12); (ii) Cognitive detachment from work is positively associated with general well-being, increased job performance and decreased psychological strain (13) and especially important for restorative sleep $(8,14-15)$. In fact, a deficient mental

1 Shared first authorship

2 Innovation Incubator, Division of Health Training Online, Leuphana University Lueneburg, Lueneburg, Germany.

3 Philipps University Marburg, Marburg, Germany.

4 Department of Clinical Psychology and Psychotherapy, University Erlangen-Nuremberg, Erlangen, Germany

5 Faculty of Psychology and Education, VU University Amsterdam, BT Amsterdam, The Netherlands.

Correspondence to: Hanne Thiart; Innovation Incubator, Division of Health Training Online, Leuphana University Lueneburg, Rotenbleicher Weg 67, 21335 Lueneburg, Germany. [Email: thiart@inkubator.leuphana.de] 
detachment has been found to mediate the negative relation between work stress and insomnia complaints (16-17); and (iii) Recreational activities are one way of fostering psychological detachment and restorative sleep by abstracting the mind from work-related thoughts $(9$, 11). In addition, engaging in leisure activities rebuilds regulatory and affective resources (18).

Evidence-based interventions are available that foster each of these three core components separately. For example cognitive behavioral therapy for insomnia (CBT-I) effectively improves sleep (19-20) and behavior activation interventions increase recreational activities (21). Third, as Querstret and Cropley (22) showed in a meta-analytic review, effective interventions exist for reducing rumination and worrying. An attempt was made to tailor such interventions to employees, and a training mainly focusing on psychological detachment from work was successfully investigated in a controlled but not randomized trial showing moderate effect sizes (23).

To the best of our knowledge, occupational health interventions that aim to promote all three core elements of recovery in an integrated manner have not yet been developed and evaluated. Integrating methods that foster all three components in one intervention and focusing it on the specific target group of stressed employees may increase the potential of inducing effective behavioral change (24-25).

Internet-based interventions for common mental and substance-use disorders as well as insomnia are wellstudied in community and clinical samples (eg, 26-27). But less is known regarding their effectiveness in occupational settings among stressed employees (28). Few studies have been effective in reducing stress and depression in workplace settings (eg, 29). Although research on internet-based interventions for workplace settings remains in its infancy, these interventions may offer some advantages to employees such as anonymity and an easy access independent of time and place. Web-based interventions may attract people who do not utilize traditional mental health services (30).

We therefore developed an internet intervention specifically targeted at employees with the aim of overcoming their deficient recovery from work and decreasing their sleep problems. The intervention (GET.ON Recovery) is based on methods to foster the aforementioned three components of successful recovery from work. We hypothesize that GET.ON Recovery will lead to a greater reduction in insomnia complaints at post-test and 6-month follow up compared to a-wait-list control (WLC) condition.

\section{Methods}

\section{Design and timeframe}

This study was a randomized controlled trial (RCT) comparing an internet-based, guided intervention group (IG) with a WLC group. Outcomes were measured pre-treatment, post-treatment (eight weeks) and at a six-month follow-up (figure 1). The study followed the CONSORT guidelines (31).

Based on the heterogeneous effects observed in the meta-analysis of Cheng (26), and taking into account that the intervention was newly developed, we expected an effect of medium size (Cohen's d $=0.50$ ). Accordingly, a sample size of $\mathrm{N}=128$ was required to detect a difference between the IG and WLC groups at post-test on the primary outcome measure (insomnia severity). This estimate was based on intention-to-treat analyses with $\alpha=0.05$ and $1-\beta=0.80$ in a two-tailed test.

Interested subjects were included and randomized from March-September 2013. Randomization was performed by an independent researcher using a computer program (randlist) that automatically assigned a 0 or 1 to each ID number, indicating allocation to either IG or WLC group. This random assignment was made at a 1:1 ratio. Randomization was not blinded.

The Psychological Ethics Committee of the Philipps University of Marburg approved the study (Nr. 2013-01K), which was registered as DRKS00004700 in the German Clinical Trial Register. Design and procedure of this study have been described in detail in the study protocol (32).

\section{Procedure}

Participants were recruited using email distribution lists to primary, secondary, and vocational schools, provided by the Ministry of Education in the German state of Nordrhein-Westfalen. Interested teachers applied to participate by an email to the primary study investigator (first author). The primary study investigator provided interested teachers with detailed information and a request to complete an online screening questionnaire. Teachers obtained access to the homepage using their email addresses and a self-chosen password. Participants who scored positive on the screening test were provided with an ID number and asked to (i) provide informed consent, (ii) complete baseline questionnaires (including a 7-day sleep diary) and (iii) participate in a diagnostic interview over the telephone. After the diagnostic interview, participants were randomized.

\section{Inclusion and exclusion criteria}

Inclusion criteria included (i) currently holding a position as a primary, secondary or vocational school teacher and working; (ii) being over the age of 18; (iii) experiencing significant clinical insomnia symptoms as measured by a score of $\geq 15$ (33) on the Insomnia Severity Index (ISI) (34); (iv) experiencing work-related rumination as measured by a score of $\geq 15$ on the cognitive 


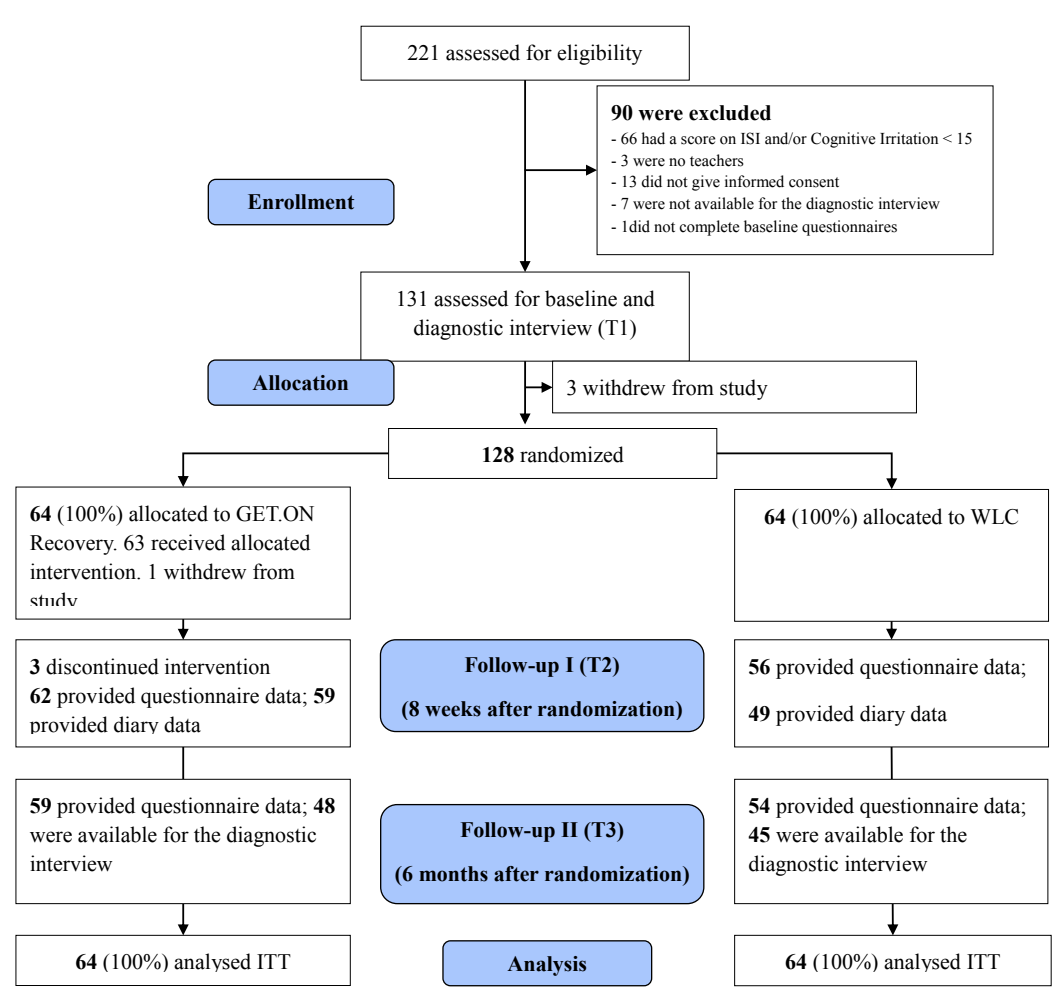

Figure 1. Flow of study participants. [WLC=waitlist control; ITT=intention to treat] irritation subscale of the Irritation Scale (IS) (35); and (v) having access to the Internet, an email address and a telephone number.

Exclusion criteria were: (i) receiving psychological help for insomnia and (ii) showing suicidal ideation based on item 9 of the Beck Depression Inventory II (BDI II) (36). Participants with scores of 2 ("I feel I would be better off dead") and 3 ("I would kill myself if I could") were excluded and received information via email regarding appropriate help. People taking sleep medication were not excluded from the study but were requested to keep their medication constant during the study period.

\section{Intervention}

GET.ON Recovery (32) uses already available and wellestablished therapeutic techniques combined in order to foster recovery from work. We used CBT-I methods, such as sleep restriction, stimulus control, sleep hygiene and cognitive interventions (37), supplemented by techniques from behavioral activation, metacognitive therapy (MCT) (38), gratitude research (39) and research on boundary management (40).

The intervention comprises six 1-week modules. In the first session, participants learn how sleep, psychological detachment and recreational activities are interconnected, and they make a plan on what sleep hygiene rules and recreational activities they plan to perform in the following week. Also, they are introduced to the 10-item online recovery diary, which is not a standard sleep diary, as recommended by Carney et al (41). The diary is tailored to the intervention and target group while covering important sleep items like time in bed or total sleep time, in order to be able to calculate and monitor sleep efficiency. In session two, participants are introduced to stimulus control and sleep restriction and plan the first steps by rescheduling their sleep for the following week. In session three, participants reflect on their progress and then continue with their sleep restriction plan. Also, they learn about behaviors which help to initiate closing time ('boundary tactics') (40) and foster detachment and sleep (gratitude journal) (38). In session four, participants learn how attention works and why worrying and rumination can be so persistent. The fifth session is based on MCT (38), teaching participants how continuous attention on ruminating thoughts can further increase their frequency and intensity.

In every session, homework includes planning of recreational activities into daily life, monitoring sleep efficiency and continuing with sleep hygiene and restriction. Additionally, participants will be asked to choose one exercise from the current session to apply throughout the forthcoming week. The intervention will conclude with a summary session (session six), which aims at preparing for difficult times when sleep gets significantly worse.

\section{Support}

The IG participants were supported by trained coaches 
according to a manual written by the first author to ensure a standardized procedure of coaching. The manual is available upon request. All communication between the coach and study participants occurred via an email messaging system on the secure training homepage. The support comprised brief, motivational, weekly feedback on exercises in completed modules. Additionally, coaches were available for questions that occurred. Coaches also provided reminders if participants did not complete a module within one week. To maximize the comparability of the participants and maintain the guidance at a minimal level, coaches were advised that the total amount of time spent on each participant should not exceed three hours for the duration of the intervention. A clinical psychologist supervised the coaches.

\section{Primary outcome measure}

Insomnia severity. Insomnia severity was measured with the German version (Pillmann, F, unpublished) of the ISI $(33,34,42)$. This 7 -item instrument is answered on a 5-point Likert scale. The total score ranges from $0-28$. It has been validated as a web-based measure as well (43) and is frequently used in iCBT-I research [for example (44)]. At screening, we used the cut-off score of $\geq 15$ indicating clinical insomnia (33). Internal consistency was previously reported to be $\alpha=0.90$ (45). In our sample, internal consistency was $\alpha=0.91$.

\section{Secondary outcome measures}

Recuperation in sleep. We used the recuperation in Sleep subscale, which is part of a well-validated $(\alpha=0.90)$ questionnaire (46) recommended as an outcome measure by the German Society of Sleep Medicine. Seven items (eg, "How relaxed did you feel upon waking up?") were answered on a 5-point Likert scale. Internal consistency in the present sample was $\alpha=0.91$

Sleep efficiency. For seven days, at T1 (after the baseline questionnaires and before the telephone interview) and $\mathrm{T} 2$, participants completed an online diary. In this diary, participants recorded the time at which they left their beds each morning, their previous evening's bedtime and their total hours of sleep (41). Sleep efficiency was computed using these data using the formula: (total hours of sleep/ time spent in bed).

Diagnoses of primary insomnia, major depression and generalized anxiety disorder. At baseline and 6-month follow up, trained clinical psychologists conducted diagnostic interviews over the telephone. Interviews provided diagnostic data on episodes of primary insomnia according to the Structured Interview for Sleep Disorders (SIS-D) (47). Diagnostic criteria used were: (i) having problems initiating, maintaining or finding restful sleep for $\geq 1$ month and $\geq 3$ nights per week, (ii) which cause clinically significant distress or impaired functioning, (iii) which do not exclusively appear in the context of hyper-, parasomnia or another mental disorder and (iv) cannot be explained by substance use. Because insomnia and depression often coexist and deficient psychological detachment is a core symptom of general anxiety disorder, these disorders were also assessed by employing the according sections of the Structured Clinical Interview for DSM-IV (SKID-I) (48).

Worrying. We measured worrying with a 3-item short version of the Penn State Worry Questionnaire (PSWQ) (49-50). For use as an outcome measure, we adapted a past-week version of the PSWQ according to the three respective German items of the PSWQ-past week (51). Internal consistency of the English ultra-brief version of the PSWQ was previously reported to be $\alpha=0.85$. In our sample, the scale displayed similar good internal consistency of $\alpha=0.88$.

Work-related rumination. We measured work-related rumination with the cognitive irritation subscale of the Irritation Scale (IS), a questionnaire which assesses occupational strain (35). We chose a score of $>14$ as a screening criterion because a score of $\geq 15$ indicates an above-average level of rumination (Stanine $\geq 7$ ) (35). Responses to the three items (eg, "even at home I have to think about problems at work") are on a 7-point Likert. The scale has good internal consistency $(\alpha=0.86)$, which also holds for our sample $(\alpha=0.87)$.

Recovery experiences. The 16-item Recovery Experience Questionnaire (REQ) (52) has been validated and includes four factors that represent four different recovery experiences: (i) psychological detachment (eg, "During time after work, I don't think of my work at all") $(\alpha=0.85)$, (ii) relaxation (eg, During time after work, I kick back and relax") ( $\alpha=0.85)$, mastery (eg, "During time after work, I do things that are challenging") $(\alpha=0.85)$, and control (eg, "During time after work, I decide myself what I do") $(\alpha=0.85)$. The questionnaire is answered on a 5-point Likert scale (not true at all, a little bit true, moderately true, mostly true, true). For our sample, internal consistency ranged from $\alpha=0.90$ (mastery) to $\alpha=0.93$ (control).

Recovery activities. The Recreation Experience and Activity Questionnaire (ReaQ) (53) rates participants' frequency of recreational activities during the past week $(0,1,2,3$, or $\geq 4$ times) for 21 items (eg, "Over the last week, I calmly enjoyed a coffee/tee"). The internal consistency of the questionnaire was $\alpha=0.88$ for our sample. 
Absenteeism. Absence from work due to sickness was measured at 6-months follow-up as the self-rated amount of total days on sick leave during the past three months.

Presenteeism. Presenteeism was measured at 6-months follow-up as the self-rated amount of total days with impaired productivity due to work-related sleep problems during the past three months.

User satisfaction. We assessed user satisfaction using a self-designed questionnaire with 8 items (eg, "I would recommend this training to a friend in need of similar help") based on the German version of the Client Satisfaction Questionnaire (CSQ-8) (54-55), answered on a 4-point Likert scale (not true at all, rather untrue, somewhat true, completely true). Internal consistency was $\alpha=0.92$.

\section{Statistical analysis}

We present the results from intention-to-treat (ITT) analyses performed with SPSS, version 21 (IBM Corp, Armonk. NY, USA). Reported P-values are two-sided with a significance level of 0.05 .

Missing data. All of the participants completed the baseline assessment. A Markov Chain Monte Carlo multivariate imputation algorithm (SPSS 21) with ten estimations per missing value was used to replace any missing T2 and T3 data (56). In multiple imputation (MI), predictors are defined which lead to estimations for the missing values. We used all pre-, post-, and 6-month follow-up values of all outcome measures as well as age and gender as predictors. At post-treatment, 7.8\% ( $N=8$ in the IG and $N=2$ in the WLC) of the data had to be replaced. At 6-month follow-up, it was 7.2\% (N=5 IG and $\mathrm{N}=10 \mathrm{WLC}$ ) for the questionnaires. For the diary data (sleep efficiency), $15 \%$ had to be replaced ( $\mathrm{N}=5$ and $\mathrm{N}=15$ in the IG and WLC, respectively). Compared to other imputation techniques, MI minimally changes variance of data, thus providing the best estimates of missing data, at least until $50 \%$ of missing data (56).

Treatment efficacy. To assess treatment efficacy, the GET.ON Recovery and WLC groups were compared on all self-report outcome measures at $\mathrm{T} 2$ using univariate analyses of covariance (ANCOVA) with T1 scores as covariates to prevent bias and increase statistical power (57-59). To assess the stability of treatment efficacy, we also conducted ANCOVA with outcome scores at T3 and baseline scores (T1) as covariates. We report P-values and Cohen's d for the between-group effect size (60) and corresponding $95 \%$ confidence intervals $(95 \% \mathrm{CI})$.

Reliable change. To enable an interpretation of clinical significance, Jacobson and Truax's (61) widely used method was employed to detect reliable changes in the primary outcome (insomnia severity) at post-treatment. A reliable change for one participant was defined if the ISI score declined from baseline to post-assessment with a reliable change index $>1.96$ (5.01 points in the ISI).

Symptom-free status. The number of participants who were free of insomnia symptoms was counted in both conditions. A score $<8$ on the ISI indicates a symptomfree status as defined by Morin (45).

Reduction of diagnosis of primary insomnia. Participants who fulfilled the diagnostic criteria of primary insomnia according to the SIS-D at baseline were of interest. The numbers of participants were calculated that maintained their diagnostic status or did not meet the criteria of primary insomnia any longer at the 6-month follow-up. We conducted Pearson's chi ${ }^{2}$ test to compare the WLC and GET.ON Recovery groups with regard to the maintenance or change to diagnose-free status of primary insomnia during the preceding 6 months. The frequency of episodes was omitted; analyses were only processed in terms of whether $(=1)$ or not $(=0)$ an episode occurred during the preceding 6 months.

Additionally, number needed to treat (MI) and corresponding $95 \%$ CI were calculated. NNT is an effect size that reflects clinical significance by comparing control and intervention groups on the frequency of a positive outcome (62). We calculated NNT by comparing the intervention and WLC groups with regard to the number of participants with or without (i) reliable change, (ii) symptom-free status and (iii) change from diagnosis of primary insomnia to diagnosis-free status.

\section{Results}

\section{Participants}

Figure 1 shows the flow of participants including those who were excluded. After screening, 90 participants were excluded: most $(\mathrm{N}=66)$ because of a score of $<15$ on the ISI and/or the cognitive irritation subscale. A total of 128 participants were randomized to either the IG $(\mathrm{N}=64)$ or the WLC group $(\mathrm{N}=64)$.

\section{Missing data}

Baseline data were available for all participants. The study attrition rate was low: $7.8 \%$ at post-treatment $(\mathrm{N}=8$ in the IG and $\mathrm{N}=2$ in the WLC) and $7.2 \%$ at the 6-month follow-up (N=5 IG and N=10 WLC), for the questionnaires. 


\section{Baseline characteristics}

The baseline characteristics of the study participants are presented in table 1 . The sample comprised 128 teachers, most of whom were female (74.2\%), with an average age of $48(\mathrm{SD}=9.9)$ years. Of these, 59 participants $(46.1 \%)$ had previous experience with a health training and/or psychotherapy, $100(78.1 \%)$ were diagnosed with primary insomnia, 18 (17.2\%) fulfilled the criteria of major depressive disorder, and 12 (9.4\%) of generalized anxiety disorder (table 1). Overall, this indicated that participants were highly affected by various mental health symptoms.

\section{Intervention usage and user satisfaction}

All of the participants $(\mathrm{N}=64)$ in the IG completed the first three modules of GET.ON Recovery. Two subjects dropped out after the third module, and one dropped out after the fourth. Reasons reported were "lack of motivation" and "lack of time". Overall, 61 (95.3\%) participants completed all six sessions. User satisfaction was high: $91 \%$ would recommend the training to a friend in need (item 4, answers of "rather yes" or "yes, completely").

\section{Primary outcome analysis - insomnia severity}

Treatment efficacy. The GET.ON Recovery group reported significantly lower insomnia severity at post-treatment than the WLC group [F=74.11, $\mathrm{P}<0.001, \mathrm{~d}=1.45(95 \% \mathrm{CI}$ 1.06-1.84)]. Sensitivity analysis (two-way ANOVA, group $x$ time interaction) led to similar results as the ANCOVA $(\mathrm{F}=63.59, \mathrm{P}<0.001)$. Table 2 shows the means and $\mathrm{SD}$ for all outcome measures separately for baseline (T1), post-treatment (T2) and 6-months follow-up (T3). Figure 2 displays the comparison of both groups on the primary outcome. Table 3 displays the results of the ANCOVA for all outcome measures separately for $\mathrm{T} 2$ and $\mathrm{T} 3$.

Reliable change and symptom-free status. There were more participants in the IG [51 (79.7\%)] with reliable improvement at post-treatment in insomnia severity than in the WLC group [18 (28.1\%)]. This finding corresponds to a NNT to achieve reliable improvement from baseline to post-treatment of 1.73 (95\% CI 1.39-2.29). Unlike four participants in the WLC, no IG participant experienced reliable deterioration ( $\mathrm{T} 2$ score of $>5.01$ above the $\mathrm{T} 1$ score).

In the GET.ON Recovery group, 23 participants $(35.9 \%)$ were symptom-free at post-treatment as indicated by a score $<8$ in the ISI. In the WLC group, 4 participants $(6.3 \%)$ were symptom free. This finding corresponds to a NNT of 3.37 (95\% CI 2.33-6.05).

\section{Secondary outcome analysis}

As shown in table 3, the ANCOVA of nearly all outcome measures showed significant differences between the two groups. Participants in the IG reported significantly less insomnia severity, rumination, and worrying at post-treatment than WLC participants. In addition, the GET.ON Recovery group showed significantly higher scores with regard to sleep efficiency, restorative sleep, recreational activities, and recovery experiences. The mastery subscale of the REQ was the only measure on which the IG and the WLC group not differ. Effect sizes ranged from $d=-0.34(95 \%$ CI $-0.69-0.01)$ (REQ control subscale) to $\mathrm{d}=-0.77(95 \% \mathrm{CI}-1.13--0.41)$ (recuperation in sleep).

\section{Long-term efficacy}

The results at $\mathrm{T} 3$ were similar to those at $\mathrm{T} 2$. ANCOVA comparing WLC and IG at T3 also showed a large effect size with regard to the primary outcome [d=1.43 (95\% CI 1.04-1.82)]. Similarly, for the IG, 27 participants $(42.2 \%)$ were symptom-free at 6 -months follow-up as indicated by a score $<8$ in the ISI. In the WLC group, 4 participants $(6.3 \%)$ were symptom free. This finding corresponds to a NNT of $2.78(95 \%$ CI 2.02-4.45).

The effect sizes of the analyses of the secondary outcomes ranged from $d=-0.34(95 \%$ CI $-0.69-0.01)$

Table 1. Demographic characteristics: means/counts, standard deviations/percentages at baseline. [WLC=waitlist control; SD=standard deviation]

\begin{tabular}{|c|c|c|c|c|c|c|c|c|c|c|c|c|}
\hline & \multicolumn{4}{|c|}{ All $(\mathrm{N}=128)$} & \multicolumn{4}{|c|}{ GET.ON Recovery $(\mathrm{N}=64)$} & \multicolumn{4}{|c|}{ WLC $(N=64)$} \\
\hline & $\mathrm{N}$ & $\%$ & Mean & SD & $\mathrm{N}$ & $\%$ & Mean & SD & $\mathrm{N}$ & $\%$ & Mean & SD \\
\hline Age & & & 48.0 & 9.9 & & & 48.4 & 9.9 & & & 47.6 & 9.9 \\
\hline Years of occupational experience & & & 19.6 & 10.8 & & & 20.4 & 10.4 & & & 18.9 & 11.1 \\
\hline Females & 95 & 74.2 & 19.0 & & 43 & 67.2 & & & 52 & 81.3 & & \\
\hline Married/partnership & 92 & 71.9 & & & 47 & 73.4 & & & 45 & 70.3 & & \\
\hline Experiences with occupational mental health trainings & 13 & 10.2 & & & 4 & 6.3 & & & 9 & 14.1 & & \\
\hline Experiences with psychotherapy & 46 & 35.9 & & & 18 & 28.1 & & & 28 & 43.8 & & \\
\hline Diagnosis of primary insomnia & 100 & 78.1 & & & 48 & 75.0 & & & 52 & 81.3 & & \\
\hline Diagnosis of major depressive disorder & 18 & 14.1 & & & 11 & 17.2 & & & 7 & 10.9 & & \\
\hline Diagnosis of generalized anxiety disorder & 12 & 9.4 & & & 6 & 9.4 & & & 6 & 9.4 & & \\
\hline
\end{tabular}


Table 2. Means and standard deviations of all outcome variables at baseline, post-treatment and six-month follow-up (intention-to-treat sample, $\mathrm{N}=128$ ). [WLC=waitlist control; $\mathrm{SD}=$ standard deviation; REQ=Recovery Experience Questionnaire]

\begin{tabular}{|c|c|c|c|c|c|c|c|c|c|c|c|c|}
\hline \multirow[t]{3}{*}{ Outcome } & \multicolumn{4}{|c|}{$\mathrm{T} 1$} & \multicolumn{4}{|c|}{ T2 } & \multicolumn{4}{|c|}{ T3 } \\
\hline & \multicolumn{2}{|c|}{ GET.ON Recovery } & \multicolumn{2}{|c|}{ WLC } & \multicolumn{2}{|c|}{ GET.ON Recovery } & \multicolumn{2}{|c|}{ WLC } & \multicolumn{2}{|c|}{ GET.ON Recovery } & \multicolumn{2}{|c|}{ WLC } \\
\hline & Mean & SD & Mean & SD & Mean & SD & Mean & SD & Mean & SD & Mean & SD \\
\hline Insomnia severity a & 18.00 & 3.24 & 17.84 & 3.17 & 9.20 & 4.55 & 15.64 & 4.31 & 8.71 & 4.71 & 15.20 & 4.39 \\
\hline Sleep efficiency ${ }^{\mathrm{b}}$ & 0.76 & 0.12 & 0.79 & 0.10 & 0.86 & 0.09 & 0.82 & 0.08 & & & & \\
\hline Recuperation in sleep ${ }^{c}$ & 2.27 & 0.62 & 2.44 & 0.80 & 3.01 & 0.75 & 2.47 & 0.65 & 3.09 & 0.74 & 2.50 & 0.65 \\
\hline Work-related rumination ${ }^{d}$ & 18.38 & 2.16 & 18.24 & 2.36 & 13.59 & 4.41 & 16.42 & 3.26 & 12.55 & 4.74 & 16.59 & 3.31 \\
\hline Worrying e & 10.63 & 4.35 & 10.16 & 3.73 & 6.19 & 3.84 & 9.21 & 4.23 & 5.42 & 3.54 & 8.72 & 4.25 \\
\hline REQ_control ${ }^{\mathrm{f}}$ & 3.03 & 1.01 & 3.01 & 0.94 & 3.41 & 0.96 & 3.10 & 0.85 & 3.43 & 0.88 & 3.10 & 0.80 \\
\hline REQ_mastery ${ }^{\dagger}$ & 2.52 & 0.76 & 2.57 & 0.85 & 2.73 & 0.84 & 2.73 & 0.85 & 2.63 & 0.69 & 2.67 & 0.78 \\
\hline REQ_psychological detachment ${ }^{\dagger}$ & 1.84 & 0.63 & 1.83 & 0.60 & 2.58 & 0.82 & 2.10 & 0.67 & 2.73 & 0.85 & 2.12 & 0.73 \\
\hline REQ_relaxation ${ }^{\dagger}$ & 2.67 & 0.82 & 2.70 & 0.80 & 3.18 & 0.80 & 2.85 & 0.76 & 3.32 & 0.79 & 2.78 & 0.71 \\
\hline Recreational activities ${ }^{g}$ & 50.78 & 14.79 & 51.40 & 11.66 & 59.16 & 13.52 & 51.64 & 12.27 & 57.41 & 12.35 & 52.39 & 13.56 \\
\hline Absenteeism ${ }^{\mathrm{h}}$ & 5.56 & 9.95 & 5.22 & 9.43 & & & & & 2.42 & 5.52 & 3.42 & 11.62 \\
\hline Presenteeism ${ }^{\mathrm{h}}$ & 14.85 & 14.51 & 13.77 & 13.94 & & & & & 7.58 & 9.94 & 11.72 & 13.90 \\
\hline
\end{tabular}

a Insomnia Severity Index, range 0-28, higher scores indicate higher insomnia severity.

b Diary data, range $0-1$, higher scores indicate increased sleep efficiency.

c Range 1-5, higher scores indicate increased recuperation.

d Irritation Scale, subscale cognitive irritation, range 3-21, higher scores indicate increased rumination.

e Penn State Worrying Questionnaire, range $0-18$, higher scores indicate increased worrying.

${ }^{\dagger}$ Subscale of REQ, range 1-5, higher scores indicated increased control/mastery/detachment/relaxation.

${ }_{9}^{9}$ Recreation Activities and Experiences Questionnaire, range 0-84, higher scores indicate increased frequency of activities.

${ }^{\mathrm{h}}$ One item, days with regard to the last three months, range 0-66, higher scores indicate more days of absenteeism/presenteeism.

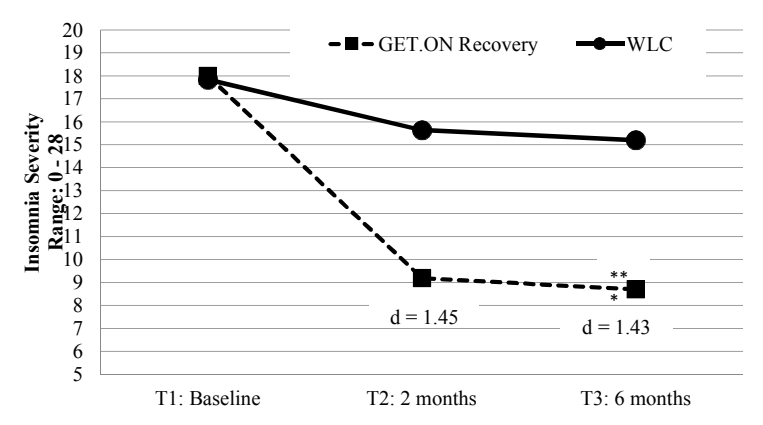

Figure 2. Comparison of intervention group (IG) and waitlist control (WLC) group on development of insomnia severity from baseline to post to 6 months-follow up. ${ }^{* * *} \mathrm{P}<0.001$ for the comparison between IG and WLC at post-treatment and at 6-months follow-up.

for recovery activities to $\mathrm{d}=0.99$ (95\% CI $0.62-1.36)$ for work-related rumination (table 3 ). IG and WLC did not differ in days of absenteeism.

\section{Change to insomnia diagnosis-free status}

Of the 128 participants, 93 (72.6\%) agreed to a diagnostic telephone interview at 6-months follow-up (figure 1). Missing values were imputed using MI. Divided almost equally between the WLC group and IG, 100 participants fulfilled the diagnostic criteria of primary insomnia at baseline (52 (81.3\%) and $48(75.0 \%)$, respectively). At 6-months follow-up, $31(64,6 \%)$ participants in IG and $17(32,7 \%)$ the WLC group were free of primary insomnia. There was a significant greater reduction in the intervention group $\left[\chi^{2}(1, \mathrm{~N}=100)=10.17, \mathrm{P}<0.05\right]$ corresponding to a NNT of 3.14 (95\% CI 1.98-7.52). Sensitivity analyses led to similar results: When missing $\mathrm{T} 3$ diagnoses were imputed using the conservative first observation carried forward (FOCF) approach, the IG also had a significantly greater reduction of primary insomnia $\left[\chi^{2}(1, \mathrm{~N}=100)=5.69, \mathrm{P}<0.05 ; \mathrm{NNT}=4.64(95 \%\right.$ CI 2.42-22.10)].

\section{Discussion}

The present study evaluated the efficacy of an internetbased guided recovery training for employees with work stress and sleep problems. Results showed that the intervention was more effective in reducing insomnia severity than the WLC condition with a large effect size $(\mathrm{d}=1.45)$. The $\mathrm{IG}$ also improved significantly more in expert-rated reduction of primary insomnia and selfrated secondary outcomes than the WLC group. In general, we observed small (presenteeism), medium (eg, sleep efficiency) and large (eg, recuperation in sleep) effect sizes for the secondary outcomes. The effects of the intervention on both primary and secondary outcomes were similar at the 6-month follow up. One possible reason for the lack of improvement for absenteeism may be that the reported number of sick days were already small at baseline not leaving much room for improvement.

Notably in this study, nearly $90 \%$ of the participants participated in an occupational health training for the first 
Table 3. Differences between groups at $\mathrm{T} 2$ and $\mathrm{T} 3$, intention-to-treat $(\mathrm{N}=128)$. [95\% $\mathrm{Cl}=95 \%$ confidence interval; ANCOVA=analysis of covariance; $R E Q=R e c o v e r y$ Experience Questionnaire

\begin{tabular}{|c|c|c|c|c|c|c|c|c|}
\hline \multirow[t]{3}{*}{ Outcome } & \multicolumn{4}{|c|}{ Between-groups effect T2 a } & \multicolumn{4}{|c|}{ Between-groups effect T3 a } \\
\hline & \multirow[t]{2}{*}{ Cohen's d } & \multirow[t]{2}{*}{$95 \% \mathrm{Cl}$} & \multicolumn{2}{|c|}{ ANCOVA $^{b}$} & \multirow[t]{2}{*}{ Cohen's d } & \multirow[t]{2}{*}{$95 \% \mathrm{Cl}$} & \multicolumn{2}{|c|}{ ANCOVA ${ }^{b}$} \\
\hline & & & $\mathrm{F}^{\mathrm{c}}$ & P-value & & & $\mathrm{F}^{\mathrm{c}}$ & P-value \\
\hline Insomnia severity & 1.45 & $1.06-1.84$ & 74.11 & $<0.001$ & 1.43 & $1.04-1.82$ & 72.65 & $<0.001$ \\
\hline Sleep efficiency & -0.47 & $-0.82--0.12$ & 18.30 & $<0.001$ & & & & \\
\hline Recuperation in sleep & -0.77 & $-1.13--0.41$ & 46.32 & $<0.001$ & -0.85 & $-1.21--0.49$ & 42.88 & $<0.001$ \\
\hline Work-related rumination & 0.73 & $0.37-1.09$ & 22.92 & $<0.001$ & 0.99 & $0.62-1.36$ & 42.94 & $<0.001$ \\
\hline Worrying & 0.75 & $0.39-1.11$ & 27.56 & $<0.001$ & .84 & $0.48-1.20$ & 29.84 & $<0.001$ \\
\hline REQ_control & -0.34 & $-0.69-0.01$ & 6.76 & $<0.05$ & -0.39 & $-0.74-0.04$ & 7.05 & $<0.01$ \\
\hline REQ_mastery & 0 & $-0.35-0.35$ & 0.03 & 0.868 & 0.05 & $-0.30-0.40$ & 0.07 & 0.79 \\
\hline REQ_psychological detachment & -0.64 & $-1.0--0.28$ & 14.61 & $<0.001$ & -0.77 & $-0.77--1.13$ & 21.70 & $<0.001$ \\
\hline REQ_relaxation & -0.42 & $-0.77--0.07$ & 9.38 & $<0.001$ & -0.72 & $-0.72--1.08$ & 21.66 & $<0.001$ \\
\hline Recreational activities & -0.58 & $-0.93--0.23$ & 15.17 & $<0.001$ & -0.34 & $-0.69--0.01$ & 6.96 & $<0.01$ \\
\hline Absenteeism & & & & & 0.11 & $-0.24-0.46$ & 0.70 & 0.41 \\
\hline Presenteeism & & & & & 0.35 & $0.00-0.70$ & 5.28 & $<0.05$ \\
\hline
\end{tabular}

a Missing data imputed by multiple imputation.

${ }^{\mathrm{b}}$ Controlling for pre-reatment scores (T1).

c Degrees of freedom not provided due to multiple imputation.

time. This result is consistent with other studies (63) showing that internet-based interventions can reach people not previously reached by traditional training formats. Acceptance can be thus regarded as high. This is additionally supported by the low intervention-dropout rate $(5 \%)$ and low study attrition. Intervention and study adherence was generally higher than with a traditional group face-to-face training (ie, 23) or guided and un-guided internet-based interventions targeting stress or insomnia $(29,64-65)$. Our high adherence rates may be due to the guidance in the training in combination with the personal contact in the diagnostic interviews. Both factors are supposed to foster accountability and trust and by that, adherence, (66) and both we share with the studies of Ritterband on iCBT-I $(44,67)$, which also reported high adherence rates. Another reason could be the high user satisfaction: $90 \%$ would recommend the training to a friend. This in turn could be explained by the interactivity of our training and our targeting approach, which might have fostered personal relevance and identification with our program. This is thought to enhance effectiveness as well as adherence to an intervention $(24,68)$.

Overall, the effects regarding insomnia severity and sleep efficiency are higher than those assessed in Cheng's meta-analysis (26) on the efficacy on internet-based CBT-I $(\mathrm{d}=0.86)$. One reason could be that our intervention included professional guidance while the studies included in Cheng's meta-analysis did not. It is well established that guidance increases clinical effectiveness (69).

In addition to sleep, a second core process of recovery is psychological detachment from work. Despite the large number of studies on this topic, to our knowledge, there is only one intervention study to date (23). This study showed that psychological detachment from work can be fostered by a face-to-face group training, at least in the short term. Our results add to these findings by showing the effects of an internet-based training in a RCT on recovery experiences like detachment, but also for corresponding recovery activities, for up to 6 months.

The present study has several strengths and limitations. A particular strength is the use of self- and expertrated health status. Given that studies demonstrating the effects of internet-based CBT-I (26) or interventions for improving detachment have exclusively relied on self-ratings, the use of both self- and expert-ratings in the present study significantly contributes to the present literature by enhancing the validity of the outcome assessment. In addition, this study's internal validity can be regarded as high since the study sample comprised only schoolteachers.

The strength of high internal validity is simultaneously a limitation with regard to external validity as generalizability is constrained to schoolteachers. Also, women were over-represented in our study, which restricts the validity of the study for male teachers. Plus, objective measures like actigraphy or polysomnography would have made the results stronger. In addition, we cannot draw conclusions regarding the superiority of this intervention over classic CBT-I, which was also found to be effective for a subgroup of stressed individuals (70). In addition, due to our WLC design, blinding was not possible. This and having a more active control group (eg, receiving simple sleep hygiene advice via the internet) in order to investigate if reported improvements are not solely the result of a new delivery mode or attention, would be important perspectives for further research.

In conclusion, the study shows that an internetbased recovery training may help teachers reduce their insomnia symptoms as well as a broad range of related health-outcomes such as rumination and worrying. The 
results may also be valid for employees with similar working conditions as teachers. Of special interest are those groups of employees who, like teachers, are more likely to face work-home interference (71), which is closely linked to a deficient recovery from work (72). Future research should replicate our findings with teachers, different groups of employees and different control group designs to strengthen the generalizability and robustness of our results.

\section{Acknowledgement}

This project was funded by the European Union (project number: EFRE: CCI 2007DE161PR001). Additionally, the study was funded by the Unfallkasse NordrheinWestfalen (Social accident insurance institution for the public sector in North Rhine-Westphalia).

Hanne Thiart, Matthias Berking, David Ebert, and Dirk Lehr are stakeholders of the "Institute for Online Health Trainings", a company aiming to transfer scientific knowledge related to the presented research into routine health care. The Institute was founded at the time of the final acceptation of the present paper. At the time of planning, conducting, and evaluating the study and writing the manuscript, the Institute did not yet exist.

\section{References}

1. Van Laethem M, Beckers DG, Kompier MA, Dijksterhuis A, Geurts SA. Psychosocial work characteristics and sleep quality: a systematic review of longitudinal and intervention research. Scand J Work Environ Health. 2013;39(6):535-49. http://dx.doi.org/10.5271/sjweh.3376.

2. Roth T. Insomnia: definition, prevalence, etiology, and consequences. J Clin Sleep Med. 2007;3:7-10.

3. Ohayon MM. Epidemiology of insomnia: what we know and what we still need to learn. Sleep Med Rev. 2002;6:97-111. http://dx.doi.org/10.1053/smrv.2002.0186.

4. Krämer K, Nolting HD. DAK-Gesundheitsreport 2010. Analyse der Arbeitsunfähigkeitsdaten- Schwerpunkt Schlafstörungen [DAK health report: Analyses of disability data]. Heidelberg: medhochzwei Verlag GmbH; 2010.

5. Kompier MAJ, Taris TW, van Veldhoven M. Tossing and turning - insomnia in relation to occupational stress, rumination, fatigue and well-being. Scand J Work Environ Health. 2012;38:238-46. http://dx.doi.org/10.5271/ sjweh.3263.

6. Westerlund $\mathrm{H}$, Alexanderson $\mathrm{K}$, Åkerstedt $\mathrm{T}$, Magnusson Hanson L, Theorell T, Kivimäki M. Work-related sleep disturbances and sickness absence in the Swedish working population, 1993-1999. Sleep. 2008,31:1169-779.
7. Daley M, Charles M, Morin CM, LeBlanc M, Grégoire J-P, Savard J. The economic burden of insomnia: direct and indirect costs for individuals with insomnia syndrome, insomnia symptoms, and good sleepers. Sleep. 2009;32(1):55-64.

8. Geurts SA, Sonnentag S. Recovery as an explanatory mechanism in the relation between acute stress reactions and chronic health impairment. Scand J Work Environ Health. 2006;32:482-92. http://dx.doi.org/10.5271/sjweh.1053.

9. Demerouti E, Bakker AB, Geurts SAE, Taris TW. Daily recovery from work-related effort during non-work time. In: Sonnentag S, Perreqé PL \& Ganster DC, editors. Current perspectives in job stress recovery. Research in organizational stress and well-being (Vol. 7). Bingley, United Kingdom: Emerald Group Publishing Limited; 2009. p. 85-123.

10. Sluiter JK, de Croon EM, Meijman TF, Frings-Dresen MH. Need for recovery from work related fatigue and its role in the development and prediction of subjective health complaints. Occup Environ Med. 2003;60(1):i62-70. http://dx.doi. org/10.1136/oem.60.suppl_1.i62.

11. Akerstedt T, Nilsson PM, Kecklund G: Sleep and recovery. In: Sonnentag S, Perrewé PL, Ganster DC, editors. Current perspectives on job-stress recovery: Research in occupational stress and well-being (Vol 7). Bingley, United Kingdom: Emerald Group Publishing Limited; 2009. p. 205-47.

12. Campbell SS. Effects of sleep and circadian rhythms of performance. In Smith AP \& Jones DM, editors. Handbook of human performance: Vol. 3. State and trait. London: Academic Press; 1992. p. 195-235.

13. Sonnentag S. Psychological Detachment from Work During Leisure Time. The Benefits of Mentally Disengaging from Work. Curr Dir Psychol Sci. 2012;21(2):114-8. http://dx.doi. org/10.1177/0963721411434979.

14. Åkerstedt T. Psychosocial stress and impaired sleep. Scand J Work Environ Health. 2006;32:493-501. http://dx.doi. org/10.5271/sjweh.1054.

15. Cropley M, Zijlstra F. Work and Rumination. In Handbook of Stress in the Occupations. Edited by Langan-Fox J, Cooper L. Cheltenham, UK: Edward Elgar Publishing Ltd; 2011:487503. http://dx.doi.org/10.4337/9780857931153.00061.

16. Querstret D, Cropley M. Exploring the relationship between work-related rumination, sleep quality and work-related fatigue. J Occup Health Psychol. 2012;17:341-53. http:// dx.doi.org/10.1037/a0028552.

17. Berset M, Elfering A, Lüthy S, Lüthi S, Semmer NK. Work stressors and impaired sleep: rumination as a mediator. Stress Heal. 2011;27:e71-e82. http://dx.doi.org/10.1002/smi.1337.

18. Trougakos JP, Hideg I. Momentary work recovery: The role of within-day breaks. In: Sonnentag S, Perreqé PL \& Ganster DC, editors. Current perspectives in job stress recovery. Research in organizational stress and well-being (Vol. 7). Bingley, United Kingdom: Emerald Group Publishing Limited; 2009. p. 37-84.

19. Morin CM, Culbert JP, Schwartz SM. Nonpharmacological interventions for insomnia: a meta-analysis of treatment efficacy. Am J Psychiatry. 1994;151:1172-80. http://dx.doi. org/10.1176/ajp.151.8.1172. 
20. Smith MT, Perlis ML, Park A, Smith MS, Pennington J, Giles DE, Buysse DJ. Comparative meta-analysis of pharmacotherapy and behavior therapy for persistent insomnia. Am J Psychiatry. 2002;159:5-11. http://dx.doi. org/10.1176/appi.ajp.159.1.5.

21. Cuijpers P, van Straten A, Warmerdam L. Behavioral activation treatments of depression: a meta-analysis. Clin Psychol Rev. 2007;27(3):318-26. http://dx.doi.org/10.1016/j. cpr.2006.11.001.

22. Querstret D, Cropley M. Assessing treatments used to reduce rumination and/or worry: a systematic review. Clin Psychol Rev. 2013;33(8):996-1009. http://dx.doi.org/10.1016/j. cpr.2013.08.004.

23. Hahn VC, Binnewies C, Sonnentag S, Mojza EJ. Learning how to recover from job stress: effects of a recovery training program on recovery, recovery-related self-efficacy, and wellbeing. J Occup Health Psychol. 2011;16:202-16. http://dx.doi. org/10.1037/a0022169.

24. Kreuter MW, Wray RJ. Tailored and targeted health communication: strategies for enhancing information relevance. Am J Health Behav. 2003;3:S227-32. http://dx.doi. org/10.5993/AJHB.27.1.s3.6.

25. Lagerveld SE, Blonk RW, Brenninkmeijer V, Wijngaards-de Meij L, Schaufeli WB. Work-focused treatment of common mental disorders and return to work: a comparative outcome study. J Occup Health Psychol. 2012;17(2):220-34. http:// dx.doi.org/10.1037/a0027049.

26. Cheng SK, Dizon J. Computerised cognitive behavioural therapy for insomnia: a systematic review and meta-analysis. Psychother Psychosom. 2012;81:206-16. http://dx.doi. $\operatorname{org} / 10.1159 / 000335379$.

27. Riper H, Blankers M, Hadiwijaya H, Cunningham J, Clarke S, Wiers R, Ebert D, Cuijpers P. Effectiveness of guided and unguided low-intensity internet interventions for adult alcohol misuse: a meta-analysis. PLoS One. 2014;9(6):e99912. http:// dx.doi.org/10.1371/journal.pone.0099912.

28. Heber E, Ebert DD, Lehr D, Nobis S, Berking M, Riper H. Efficacy and cost-effectiveness of a web-based and mobile stress-management intervention for employees: design of a randomized controlled trial. BMC Public Health. 2013;3:655. http://dx.doi.org/10.1186/1471-2458-13-655.

29. Ebert DD, Lehr D, Boß L, Riper H, Cuijpers P, Andersson G, Thiart H, Heber E, Berking M. Efficacy of an Internetbased problem-solving training for teachers: Results of a randomized controlled trial. Scand J Work Environ Health. 2014;40(6):582-96. http://dx.doi.org/10.5271/sjweh.3449.

30. Andersson G, Cuijpers P. Pros and cons of online cognitivebehavioural therapy. Brit J Psychiat. 2008;193:270-71. http:// dx.doi.org/10.1192/bjp.bp.108.054080.

31. Schulz KF, Altman DG, Moher D, for the CONSORT Group. CONSORT 2010 Statement: updated guidelines for reporting parallel group randomised trials. BMC Med. 2010;8:18. http://dx.doi.org/10.1186/1741-7015-8-18.

32. Thiart H, Lehr D, Ebert DD, Sieland B, Berking M, Riper H. Log in and breathe out: Efficacy and cost-effectiveness of an online sleep training for teachers affected by work-related strain - study protocol for a randomized controlled trial. Trials. 2013;14:169. http://dx.doi.org/10.1186/1745-6215-14-169.

33. Morin CM, Belleville G, Bélanger L, Ivers H. The Insomnia Severity Index: psychometric indicators to detect insomnia cases and evaluate treatment response. Sleep. 2011;34(5):6018 .

34. Bastien $\mathrm{CH}$, Vallières A, Morin CM. Validation of the insomnia severity index as an outcome measure for insomnia research. Sleep Med. 2001;2:297-307. http://dx.doi.org/10.1016/ S1389-9457(00)00065-4.

35. Mohr G, Rigotti T, Müller A. IS - Irritations-Skala zur Erfassung arbeitsbezogener Beanspruchungsfolgen [Irritation Scale for the assessment of psychological strain in work contexts]. Göttingen: Hogrefe; 2007.

36. Hautzinger M, Keller F, Kühner C. BDI-II. Beck DepressionsInventar Revision [Beck Depression Inventory Revision]. Frankfurt am Main: Harcourt Test Services; 2006.

37. Siebern AT, Manber R. Insomnia and its effective nonpharmacologic treatment. Med Clin North Am. 2010;94:58191. http://dx.doi.org/10.1016/j.mcna.2010.02.005.

38. Wells A. Metacognitive Therapy for Anxiety and Depression. New York: Guilford Press; 2009.

39. Emmons RA, McCullough ME. Counting blessings versus burdens: an experimental investigation of gratitude and subjective well-being in daily life. J Pers Soc Psychol. 2003;84:377-89. http://dx.doi.org/10.1037/00223514.84.2.377.

40. Kreiner GE, Hollensbe EC, Sheep ML. Balancing borders and bridges: negotiating the work-home interface via boundary work tactics. Acad Manage J. 2009;52:704-30. http://dx.doi. org/10.5465/AMJ.2009.43669916.

41. Carney CE, Buysse DJ, Ancoli-Israel S, Edinger JD, Krystal $\mathrm{AD}$, Lichstein KL, Morin CM. The consensus sleep diary: standardizing prospective sleep self-monitoring. Sleep. 2012;35(2):287-302.

42. Morin CM. Insomnia: Psychological Assessment and Management. New York, NY: Guilford Press; 1993.

43. Thorndike FP, Ritterband LM, Saylor DK, Magee JC, GonderFrederick LA, Morin CM. Validation of the insomnia severity index as a web-based measure. Behav Sleep Med. 2011;9:21623. http://dx.doi.org/10.1080/15402002.2011.606766.

44. Ritterband LM, Thorndike FP, Gonder-Frederick LA, Magee JC, Bailey ET, Saylor DK, Morin CM. Efficacy of an Internetbased behavioral intervention for adults with insomnia. Arch Gen Psychiatry. 2009;66:692-8. http://dx.doi.org/10.1001/ archgenpsychiatry.2009.66.

45. Morin CM, Belleville G, Bélanger L, Ivers H. The Insomnia Severity Index. psychometric indicators to detect insomnia cases and evaluate treatment response. Sleep. 2011;34(5):6018 .

46. Görtelmeyer R. SF-A/R and SF-B/R Sleep Questionnaire A and $\mathrm{B}$ (revised version). Göttingen: Hogrefe; 2011.

47. Schramm E, Hohagen F, Grasshoff U, Riemann D, Hajak G, Weeß HG, Berger M. Test-retest reliability and validity 
of the structured interview for sleep disorders according to DSM-III-R. Am J Psychiat. 1993;150:867-72. http://dx.doi. org/10.1176/ajp.150.6.867.

48. Wittchen HU, Zaudig M, Fydrich T. Strukturiertes Klinisches Interview für DSM-IV Achse I und II [Structured Clinical Interview for DSM-IV axis I and II]. Göttingen: Hogrefe; 1997.

49. Berle D, Starcevic V, Moses K, Hannan A, Milicevic D, Sammut P. Preliminary validation of an ultra-brief version of the Penn State Worry Questionnaire. Clin Psychol Psychother. 2011;18:339-46. http://dx.doi.org/10.1002/cpp.724.

50. Meyer TJ, Miller ML, Metzger RL, Borkovec TD. Development and validation of the Penn State Worry Questionnaire. Behav Res Ther. 1990;28:487-95. http:// dx.doi.org/10.1016/0005-7967(90)90135-6.

51. Stöber J, Bittencourt J: Weekly assessment of worry: an adaptation of the Penn State Worry Questionnaire for monitoring changes during treatment. Behav Res Ther. 1998; 36:645-56. http://dx.doi.org/10.1016/S00057967(98)00031-X.

52. Sonnentag S, Fritz C. The recovery experience questionnaire: development and validation of a measure assessing recuperation and unwinding at work. J Occup Health Psycho. 2007;12:204-21. http://dx.doi.org/10.1037/10768998.12.3.204.

53. Lehr D. The Recreation Experience and Activity Questionnaire (ReaQ). In: Burnout und chronischer beruflicher Stress [Burnout and chronic work-stress]. Edited by Koch S, Lehr D, Hillert A. Göttingen: Hogrefe. Forthcoming 2015.

54. Schmidt J, Lamprecht F, Wittmann WW. Satisfaction with inpatient management. Development of a questionnaire and initial validity studies. Psychother Psychosom Med Psychol. 1989;39:248-55.

55. Attkisson CC, Zwick R. The client satisfaction questionnaire. Psychometric properties and correlations with service utilization and psychotherapy outcome. Eval Program Plann. 1982;5:233-7. http://dx.doi.org/10.1016/01497189(82)90074-X.

56. Schafer JL, Graham JW. Missing data: our view of the state of the art. Psychol Methods. 2002;7(2):147-77. http://dx.doi. org/10.1037/1082-989X.7.2.147.

57. Egbewale BE, Lewis M, Sim J. Bias, precision and statistical power of analysis of covariance in the analysis of randomized trials with baseline imbalance: a simulation study. BMC Med Res Methodol. 2014;14:49. http://dx.doi.org/10.1186/14712288-14-49.

58. Van Breukelen GJ. ANCOVA versus change from baseline: more power in randomized studies, more bias in nonrandomized studies. J Clin Epidemiol. 2006;59(9):920-5. http://dx.doi. org/10.1016/j.jclinepi.2006.02.007.

59. Van Breukelen GJ, Van Dijk KR. Use of covariates in randomized controlled trials. J Int Neuropsychol Soc. 2007;13(5):903-4. http://dx.doi.org/10.1017/S1355617707071147.

60. Cohen J. Statistical Power Analysis for the Behavioural Sciences. 2nd edition. Hillsdale, NJ: Lawrence Erlbaum Associates; 1988.
61. Jacobson NS, Truax P. Clinical significance: a statistical approach to defining meaningful change in psychotherapy research. J Consult Clin Psychol. 1991;59:12-9. http://dx.doi. org/10.1037/0022-006X.59.1.12.

62. Citrome L, Ketter TA. When does a difference make a difference? Interpretation of number needed to treat, number needed to harm, and likelihood to be helped or harmed. Int J Clin Pract. 2013;67(5):407-11. http://dx.doi.org/10.1111/ ijcp.12142.

63. McKellar J, Austin J, Moos R. The first step: a review of lowintensity interventions for stepped care.Addict Sci Clin Pract. 2012;7(1):26. http://dx.doi.org/10.1186/1940-0640-7-26.

64. Ström L, Pettersson R, Andersson G. Internet-based treatment for insomnia: a controlled evaluation. J Consult Clin Psychol 2004, 72(1):113-20. http://dx.doi.org/10.1037/0022006X.72.1.113.

65. van Straten A, Emmelkamp J, de Wit J, Lancee J, Andersson G, van Someren EJ, Cuijpers P. Guided Internet-delivered cognitive behavioural treatment for insomnia: a randomized trial. Psychol Med. 2014;44(7):1521-32. http://dx.doi. org/10.1017/S0033291713002249.

66. Mohr D, Cuijpers P, Lehman K. Supportive Accountability: A Model for Providing Human Support to Enhance Adherence to eHealth Interventions. J Med Internet Res. 2011;13(1):e30. http://dx.doi.org/10.2196/jmir.1602.

67. Ritterband LM, Bailey ET, Thorndike FP, Lord HR, FarrellCarnahan L, Baum LD. Initial evaluation of an Internet intervention to improve the sleep of cancer survivors with insomnia. Psychooncology. 2012;21(7):695-705. http:// dx.doi.org/10.1002/pon.1969.

68. Donkin L, Christensen H, Naismith SL, Neal B, Hickie IB, Glozier NJ Med Internet Res. A systematic review of the impact of adherence on the effectiveness of e-therapies.2011;13(3):e52.

69. Palmqvist B, Carlbring P, Andersson G. Internet-delivered treatments with or without therapist input: does the therapist factor have implications for efficacy and cost? Expert Rev Neurother. 2007;7:291-7.

70. Jernelöv S, Lekander M, Blom K, Rydh S, Ljótson B, Axelsson J, Kaldo V. Efficacy of a behavioral self-help treatment with or without therapist guidance for co-morbid and primary insomnia -a randomized controlled trial. BMC Psychiatry. 2012;12:5. http://dx.doi.org/10.1186/1471-244X-12-5.

71. Lehr D: Belastung und Beanspruchung im Lehrerberuf: Gesundheitliche Situation und Evidenz für Risikofaktoren [Stress and strain in teachers: Health situation and evidence for risc factors]. In Handbuch der Forschung zum Lehrerberuf. Edited by Terhart E, Bennewitz H, Rothland M. Münster: Waxmann; 2011:757-73.

71. Geurts SAE, Taris TW, Kompier MAJ, Dikkers JSE, Van Hooff LM, Kinnunen UM. Work-home interaction from a work psychological perspective: Development and validation of a new questionnaire, the SWING. Work \& Stress. 2005;19(4):319 39. http://dx.doi.org/10.1080/02678370500410208.

Received for publication: 8 July 2014 